Journal of Data Science 5(2007), 315-333

\title{
Stochastic Diffusion Modeling of Degradation Data
}

\author{
Sheng-Tsaing Tseng and Chien-Yu Peng \\ National Tsing-Hua University
}

\begin{abstract}
Accelerated degradation tests (ADTs) can provide timely reliability information of product. Hence ADTs have been widely used to assess the lifetime distribution of highly reliable products. In order to properly predict the lifetime distribution, modeling the product's degradation path plays a key role in a degradation analysis. In this paper, we use a stochastic diffusion process to describe the product's degradation path and a recursive formula for the product's lifetime distribution can be obtained by using the first passage time (FPT) of its degradation path. In addition, two approximate formulas for the product's mean-time-to-failure (MTTF) and median life (B50) are given. Finally, we extend the proposed method to the case of ADT and a real LED data is used to illustrate the proposed procedure. The results demonstrate that the proposed method has a good performance for the LED lifetime prediction.
\end{abstract}

Key words: Accelerated degradation test, degradation test, first passage time, stochastic diffusion process.

\section{Introduction and Summary}

Nowadays, many products are designed and manufactured to function for a long period before they fail. Hence, determining product reliability is a great challenge to manufacturers of highly reliable products with only a relatively short period of time available for internal life testing. Although the techniques of censoring and/or accelerating the life by testing at higher levels of stress may help, they offer little help for really highly reliable products. The main reason is that it is rather difficult to obtain enough time-to-failure data to estimate product's lifetime efficiently. Under this situation, an alternative approach is to collect the "degradation" data at higher levels of stress for predicting a product's lifetime at a certain use-stress level. Such an experiment is called an accelerated degradation test (ADT).

Nelson (1990), Meeker and Escobar (1998), and Chao (1999) reviewed the degradation literature, surveyed its wide applications and described statistical methods for ADT models. Numerous models have been developed and applied 
to some specific degradation tests. Chang (1992) analyzed two-variable accelerated degradation data of a specific power supply. Tseng, Hamada and Chiao (1995) presented a case study of improving the reliability of fluorescent lamps. Lu, Park and Yang (1997) derived linear model for hot-carrier-induced degradation of metalized and oxidized semiconductors. Yu and Tseng (1998) proposed an on-line stopping procedure for determining an appropriate termination time for an ADT of light emitting diode (LED) product. Meeker, Escobar and Lu (1998) proposed mixed effects model to describe the degradation paths of an ADT and the results are applied to the device-B Power-output data. Shiau and Lin (1999) derived a nonparametric model for describing degradation of LED products. Other important research applications of degradation models are in the areas such as micro-electronic components, the laser diode, food and drugs, metals fatigue testing, plasma display panels, liquid crystal display and digital light processing projectors and other dependable systems.

The performance of an ADT, obviously, strongly depends on the appropriateness of the modeling of its degradation path. A typical degradation path consists of mean degradation curve and its error term (measurement error). There are two well-known approaches in the literature. First, the mixed effects model is one of the most popular approaches in degradation analysis (Meeker et al., 1998, and Bae and Kvam, 2004). In order to describe the unit-to-unit variations of the test units, the unknown parameters of mean degradation path were described in terms of the mixed (or random) effects. Generally speaking, to find the maximum likelihood estimates (MLEs) of the unknown parameters, the mixed effects model is computationally intensive. Although several approximate procedures have been proposed in the literature (Bae and Kvam, 2004) to release computational efforts of complex likelihood function, these procedures do not always guarantee that the precise parameter estimations can be obtained. In addition, most of the mixed effects formulations did not take the time-dependent error structure into consideration.

Due to the mentioned-above difficulties, the stochastic process formulation turned out to be an alternative approach to model the product's degradation path. Typical examples for this approach are Doksum and Hóyland (1992), Yu and Tseng (2002), Tseng, Tang and Ku (2003), and Lawless and Crowder (2004). Most literature in this area assume that the error term of degradation path follows a Wiener process (which is a time-dependent version of the iid $N(0,1)$ ) or a Gamma process. However, motivated from the residual analysis of a real LED data (see Section 6 and Figures 4-5 below), a stochastic diffusion process is more appropriate to model the error term of degradation path. Hence, a stochastic diffusion formulation is adopted in this paper.

Assuming the error term satisfies a stochastic differential equation, we show 
that the proposed degradation model turns out to be a Gauss-Markov process. Then, the product's lifetime distribution can be expressed in terms of a secondkind Volterra intergral equation. Furthermore, if the proposed degradation model satisfies a specific condition (which was motivated from LED data in Section 6), then the product's lifetime distribution reduces to be a function of truncated inverse Gaussian (TIG) distribution. Hence, two approximate formulas for the product's mean-time-to-failure (MTTF) and median life (B50) can be easily derived. Finally, we extend the proposed method to the case of ADT and a real LED data is used to illustrate the proposed procedure. The result demonstrates that the proposed method has a good performance in the LED lifetime prediction.

The rest of this paper is organized as follows. Section 2 uses a stochastic diffusion model to formulate the product's degradation path. Section 3 derives the product's lifetime distribution of the proposed procedure. Section 4 presents the approximate formulas for the product's MTTF and B50. Section 5 extends the proposed procedure to an ADT with two accelerating variables. Section 6 uses the LED data to illustrate the proposed method. A performance comparison between our proposed method and mixed effect model is also addressed. Finally, Section 7 addresses some concluding remarks.

\section{Problem Formulation}

Let $L(t)$ denote the degradation path of a specific quality characteristic of a product at time $t$. Without loss of generality, we assume that $L(t)$ is a decreasing function of time $t$ and $\omega$ denotes the critical level for this degradation path. Then the product's lifetime $Z$ can be suitably defined as the first time when $L(t)$ crosses the critical level $\omega$, that is,

$$
Z=\inf \{t \mid L(t) \leq \omega\} .
$$

Traditionally, we often use the following model to describe the degradation path:

$$
L(t)=M(t)+\varepsilon(t),
$$

where $M(t)$ and $\varepsilon(t)$ denote the mean degradation path and the error term at time $t$, respectively. Since degradation is a continuous decay process, the independence assumption among $\varepsilon(t)$ appears to be questionable (Tseng et al., 2003). With this in mind, the following stochastic differential equation is more appropriate to describe $\varepsilon(t)$ (Øksendal, 2003):

$$
d \varepsilon(t)=s(t) d B(t)
$$

where $B(t)$ denotes a standardized Brownian motion. Note that if $s(t)$ is a constant, then (2.2) reduces to degradation path in Tseng and Peng (2004). 
The goal of a degradation analysis is to find the product's lifetime distribution of $Z$ and assess it's lifetime information such as MTTF and B50. Hence, some related decision problems are summarized as follows:

(i) How to derive the probability density function of $Z$ ?

(ii) How to obtain the product's MTTF and B50?

(iii) How to extend the proposed procedure to analyze an ADT data?

We will address these questions in the following sections.

\section{The probability density function of $Z$}

From (2.2)-(2.3), $L(t)$ can be expressed as follows:

$$
L(t)=M(t)+\int_{0}^{t} s(x) d B(x) .
$$

Following from Hoel et al. (1972, pages 135-147), it is easily seen that $\{L(t) \mid t \geq 0\}$ follows a Gaussian process with the following properties:

(i) $\mathrm{E}(L(t))=M(t)$ and $\operatorname{Var}(L(t))=\int_{0}^{t} s^{2}(x) d x$;

(ii) $\mathrm{E}\left(L^{\prime}(t)\right)=M^{\prime}(t)$ and $\operatorname{Var}\left(L^{\prime}(t)\right)=s^{2}(t)$;

(iii) If $t_{1} \leq t_{2}$, then $\operatorname{Cov}\left(L\left(t_{1}\right), L\left(t_{2}\right)\right)=\int_{0}^{t_{1}} s^{2}(x) d x$.

From the result of Mehr and McFadden (1965), we have the following result:

\section{Proposition 1:}

$$
L(t)=M(t)+B\left(\int_{0}^{t} \Upsilon(x) d M(x)\right)
$$

where

$$
\Upsilon(t)=\frac{\operatorname{Var}\left(L^{\prime}(t)\right)}{\mathrm{E}\left(L^{\prime}(t)\right)}, \text { for all } t>0 .
$$

Now, set $h_{1}(t)=\int_{0}^{t} \Upsilon(x) d M(x), h_{2}(t)=1$, and $S(t)=M(t)-\omega$. Then by using Theorem 3.1 of Di Nardo, et al. (2001), the pdf of $Z, f_{Z}(t)$, satisfies the following non-singular second-kind Volterra integral equation:

$$
f_{Z}(t)=-2 \Psi(t \mid 0,0)+2 \int_{0}^{t} f_{Z}(x) \Psi(t \mid M(x)-\omega, x) d x
$$


where

$$
\Psi(t \mid y, x)=\left\{\frac{M^{\prime}(t)}{2}-\left(\frac{M(t)-\omega}{2}-\frac{y}{2}\right) \frac{\Upsilon(t) M^{\prime}(t)}{\int_{x}^{t} \Upsilon(u) d M(u)}\right\} g(t \mid y, x)
$$

and

$$
g(t \mid y, x)=\frac{1}{\sqrt{2 \pi \int_{x}^{t} \Upsilon(u) d M(u)}} \exp \left(-\frac{(M(t)-\omega-y)^{2}}{2 \int_{x}^{t} \Upsilon(u) d M(u)}\right) .
$$

Once the pdf $f_{Z}(t)$ is obtained, then the product's MTTF and $B 50$ can be solved numerically by $M T T F=\int_{0}^{\infty} t f_{Z}(t) d t$ and $\int_{0}^{B 50} f_{Z}(t) d t=0.5$. However, the above recursive formula for $f_{Z}(t)$ is complicated for practical applications.

\section{Expressions for MTTF and $B 50$ under constant $\Upsilon(t)$}

Hereafter, we restrict our attention to a special case of $\Upsilon(t)$ is a constant. This assumption is motivated from a LED real example in Section 6. From (3.3), note that $\Upsilon(t)$ is always a negative function of time $t$. We further assume that $\Upsilon(t)$ is a constant (independent of $t$ ), that is,

$$
\Upsilon(t)=-\sigma^{2},
$$

where $\sigma>0$. Under this assumption, the expression of $f_{Z}(t)$ can be further simplified as follows:

$$
f_{Z}(t)=\frac{-(1-\omega) M^{\prime}(t)}{K \sqrt{2 \pi \sigma^{2}(1-M(t))^{3}}} \exp \left(-\frac{(M(t)-\omega)^{2}}{2 \sigma^{2}(1-M(t))}\right), 0<t<\infty
$$

where $K$ is a normalizing constant.

Let

$$
Y=1-M(Z) .
$$

Then $Y$ follows a truncated inverse Gaussian (TIG) distribution. That is, the pdf of $Y$ can be expressed as (Chhikara and Folks, 1989):

$$
g_{Y}(y)=\frac{(1-\omega)}{K \sqrt{2 \pi \sigma^{2} y^{3}}} \exp \left(-\frac{(y-(1-\omega))^{2}}{2 \sigma^{2} y}\right), 0<y<1 .
$$

Here we introduce the approximate formulas for the product's MTTF $(=E(Z))$ and $B 50$, respectively. 


\subsection{Approximate formula for MTTF}

From (4.3), we have $Z=M^{-1}(1-Y)$. Hence, by using $\delta$-method, it is easy to derive the following result.

$$
\mathrm{MTTF} \approx M^{-1}(1-\mathrm{E}(Y))-\frac{\operatorname{Var}(Y)}{2} \frac{M^{\prime \prime}\left(M^{-1}(1-\mathrm{E}(Y))\right)}{\left(M^{\prime}\left(M^{-1}(1-\mathrm{E}(Y))\right)\right)^{3}},
$$

where

$$
\begin{aligned}
\mathrm{E}(Y)= & (1-\omega)\left(\frac{\Phi(\omega / \sigma)-G}{\Phi(\omega / \sigma)+G}\right) \\
\operatorname{Var}(Y)= & \sigma^{2}(1-\omega)\left(\frac{\Phi(\omega / \sigma)-G}{\Phi(\omega / \sigma)+G}\right)+(1-\omega)^{2}\left(1-\left(\frac{\Phi(\omega / \sigma)-G}{\Phi(\omega / \sigma)+G}\right)^{2}\right) \\
& \quad-2 \sigma(1-\omega)\left(\frac{\phi(\omega / \sigma)}{\Phi(\omega / \sigma)+G}\right), \\
G= & \exp \left(\frac{2(1-\omega)}{\sigma^{2}}\right) \Phi\left(\frac{\omega-2}{\sigma}\right),
\end{aligned}
$$

and $\Phi$ and $\phi$ are the cdf and pdf of $N(0,1)$, respectively. Note that $E(Y)$ and $\operatorname{Var}(Y)$ in (4.4) can be obtained directly by Patel (1965).

In practical applications, when $\sigma^{2} \rightarrow 0$, it is easily seen from l'Hospital rule that

$$
G \rightarrow 0, \quad \frac{\Phi(\omega / \sigma)-G}{\Phi(\omega / \sigma)+G} \rightarrow 1
$$

and

$$
\frac{\phi(\omega / \sigma)}{\Phi(\omega / \sigma)+G} \rightarrow 0
$$

Hence, we obtain a simple expression for MTTF as follows:

Proposition 2:

$$
\mathrm{MTTF} \approx M^{-1}(\omega)-\frac{\sigma^{2}(1-\omega)}{2} \frac{M^{\prime \prime}\left(M^{-1}(\omega)\right)}{\left(M^{\prime}\left(M^{-1}(\omega)\right)\right)^{3}} .
$$

\subsection{Approximate formula for the median life}

The product's $p$-th percentile $\left(\xi_{p}=F_{Y}^{-1}(p)\right)$ is also an important reliability measure of the product. Let

$$
X \sim I G\left(1-\omega,(1-\omega)^{2} / \sigma^{2}\right),
$$


then

$$
F_{Y}(y)=F_{X}(y) / K
$$

where

$F_{X}(y)=\Phi\left(\frac{1-\omega}{\sigma \sqrt{y}}\left(\frac{y}{1-\omega}-1\right)\right)+\exp \left(\frac{2(1-\omega)}{\sigma^{2}}\right) \Phi\left(-\frac{1-\omega}{\sigma \sqrt{y}}\left(\frac{y}{1-\omega}+1\right)\right)$.

Again, there is no closed form for $\xi_{p}$. From the approximate transformation in Whitmore and Yalovsky (1978), we obtain

$$
\frac{\sigma}{2 \sqrt{1-\omega}}+\frac{\sqrt{1-\omega}}{\sigma} \ln \left(\frac{Y}{1-\omega}\right) \stackrel{d}{\longrightarrow} N(0,1), \text { if } \sigma^{2} \rightarrow 0 .
$$

Thus,

$$
F_{X}(y) \approx \Phi\left[\frac{\sigma}{2 \sqrt{1-\omega}}+\frac{\sqrt{1-\omega}}{\sigma} \ln \left(\frac{y}{1-\omega}\right)\right] .
$$

From (4.3), we have the following result:

$$
\xi_{p} \approx M^{-1}\left[1-(1-\omega) \exp \left(\frac{\Phi^{-1}(K p) \sigma}{\sqrt{1-\omega}}-\frac{\sigma^{2}}{2(1-\omega)}\right)\right] .
$$

Hence, as $p=0.5$ and $K \approx 1$, the median life (B50) can be reduced to

Proposition 3:

$$
B 50 \approx M^{-1}\left[1-(1-\omega) \exp \left(-\frac{\sigma^{2}}{2(1-\omega)}\right)\right] .
$$

\section{Applications to Accelerated Degradation Tests}

For a highly reliable product with a slow degradation rate, we usually use higher-level stress variables (such as temperature, voltage, electric current, etc.) to accelerate the degradation path. Without loss of generality, we assume that $\left(A_{0}, T_{0}\right)$ denotes the normal use stress and $\left\{A_{i}\right\}_{i=1}^{m}$ and $\left\{T_{j}\right\}_{j=1}^{n}$ are the electric current and temperature variables, where $m$ and $n$ denote the number of stress levels for these two accelerating variables, respectively.

Let $L_{i j}(t)$ denote the degradation path under the combination of $\left(A_{i}, T_{j}\right)$. Then the degradation path in (2.2) can be modified as follows:

$$
L_{i j}(t)=M_{i j}(t)+\varepsilon_{i j}(t) .
$$

Generally speaking, without specifying the functional form of $M_{i j}(t)$ and its life-stress relation, it is impossible to extrapolate the lifetime information under 
a normal use condition. Now, motivated from Yu and Tseng (1998), we assume that

$$
M_{i j}(t)=\exp \left(-\eta_{i j} t^{\delta}\right), \forall t \geq 0,
$$

where $\eta_{i j}$ follows a generalized Eyring law, that is,

$$
\ln \eta_{i j}=\gamma_{0}+\gamma_{1} \ln \left(A_{i}\right)+\frac{\gamma_{2}}{273.15+T_{j}}+\frac{\gamma_{3} \ln \left(A_{i}\right)}{273.15+T_{j}}
$$

Note that the above formula has been widely used for describing the degradation path of the light intensity of the LED lamp (Fukuda, 1991).

Let $L_{00}(t)$ denote the degradation path under $\left(A_{0}, T_{0}\right)$. Then the product's lifetime, $Z_{00}$, can be obtained directly from $(2.1)$ by replacing $L(t)$ with $L_{00}(t)$. Let $M T T F_{00}$ and $B 50_{00}$ denote the MTTF and the median life of $Z_{00}$, respectively. Then, as $\sigma^{2} \rightarrow 0$, the product's MTTF at the use condition can be expressed as:

$$
\operatorname{MTTF}_{00} \approx\left(\frac{-\ln \omega}{\eta_{00}}\right)^{1 / \delta}\left(1+\frac{\sigma^{2}(1-\omega)(1-\delta-\delta \ln \omega)}{2 \delta^{2} \omega^{2}(\ln \omega)^{2}}\right) .
$$

Similarly, the median life can be expressed as:

$$
B 50_{00} \approx\left(\frac{-\ln \left(1-(1-\omega) \exp \left(-\sigma^{2} /(2(1-\omega))\right)\right)}{\eta_{00}}\right)^{1 / \delta} .
$$

Now, if the unknown parameters $\eta_{00}, \delta$ and $\sigma^{2}$ in (5.4) and (5.5) can be estimated successfully, then the product's predicted MTTF and estimated B50 can be easily obtained. In the following subsection, we will address this issue.

\subsection{Estimations of unknown parameters}

Extending the results of Sections 3 and 4 to the case of ADT, $L_{i j}(t)$ follows a Gauss-Markov process, hence

$$
L_{i j}(t) \stackrel{d}{\sim} N\left(M_{i j}(t), \sigma^{2}\left(1-M_{i j}(t)\right)\right)
$$

Let $L_{i j k}\left(t_{l}\right)$ denote the degradation path of $k$-th unit under $\left(A_{i}, T_{j}\right)$ at time $t_{l}$. Set $\Delta L_{i j k}\left(t_{l}\right)=L_{i j k}\left(t_{l}\right)-L_{i j k}\left(t_{l-1}\right)$ and $\Delta M_{i j}\left(t_{l}\right)=M_{i j}\left(t_{l}\right)-M_{i j}\left(t_{l-1}\right)$. For all $1 \leq k \leq n_{i j}, 1 \leq l \leq l^{*}$, then

$$
\Delta L_{i j k}\left(t_{l}\right) \stackrel{\text { ind. }}{\sim} N\left(\Delta M_{i j}\left(t_{l}\right),-\sigma^{2} \Delta M_{i j}\left(t_{l}\right)\right)
$$


By using the independent increment property, the likelihood function can be expressed as follows:

$$
\mathcal{L}\left(\gamma_{0}, \gamma_{1}, \gamma_{2}, \gamma_{3}, \delta, \sigma\right)=\prod_{(i, j)} \prod_{k=1}^{n_{i j}} \prod_{l=1}^{l^{*}}\left\{\frac{1}{\sigma \sqrt{-\Delta M_{i j}\left(t_{l}\right)}} \phi\left(\frac{\Delta L_{i j k}\left(t_{l}\right)-\Delta M_{i j}\left(t_{l}\right)}{\sigma \sqrt{-\Delta M_{i j}\left(t_{l}\right)}}\right)\right\} .
$$

Hence, the MLE for all unknown parameters can be solved by maximizing the above likelihood function.

\section{Analysis of LED Degradation Data}

LED products have become widely used in a variety of fields with applications ranging from consumer electronics to optical fiber transmission systems. High reliability is especially required in optical fiber transmission. Hence, obtaining lifetime information (or distributions) for high-reliability components is a challenge to the manufacturers. For such a highly reliable product, an ADT is used to speed up its degradation. From engineering knowledge, electric current and temperature are two suitable accelerating variables for LED products. Three higher stress levels for electric current (say, $A_{1}=10 \mathrm{~mA}, A_{2}=20$ $\mathrm{mA}$ and $A_{3}=30 \mathrm{~mA}$ ) and for temperature (say, $T_{1}=25^{\circ} \mathrm{C}, T_{2}=65^{\circ} \mathrm{C}$ and $T_{3}=105^{\circ} \mathrm{C}$ ) are carefully chosen. Due to the cost consideration, only six combinations (cells) of these two accelerating variables, say $\left\{\left(A_{i}, T_{j}\right)\right\},(i, j) \in$ $\{(1,1),(1,2),(1,3),(2,1),(3,1),(3,3)\}$, are conducted and the test units for these cells are $\left(n_{11}, n_{12}, n_{13}, n_{21}, n_{31}, n_{33}\right)=(24,25,24,25,25,22)$.

A key quality characteristic of LED lamp is its light intensity. LED light intensity degrades over time. The measuring frequency of its light intensity is 168 hours (except for few cases) and the experiment was terminated at the time of 9998 hours for each cell. Hence, 58 observations of light intensity of LED products are recorded ${ }^{1}$. Figure 1 shows the plots of $\left\{L_{i j k}(t)\right\}_{k=1}^{n_{i j}}$, for all $(i, j)$. The goal of this ADT is to assess the lifetime distribution of LED lamp under a normal use conditions, say $A_{0}=7.5 \mathrm{~mA}$, and $T_{0}=20^{\circ} \mathrm{C}$. Note that the degradation paths in the cell of $\left(30 \mathrm{~mA}, 105^{\circ} \mathrm{C}\right)$ were designed to estimate the interaction effect between temperature and current.

\footnotetext{
${ }^{1}$ The readers who are interested in this data set may send email to the author via sttseng@stat.nthu.edu.tw
} 


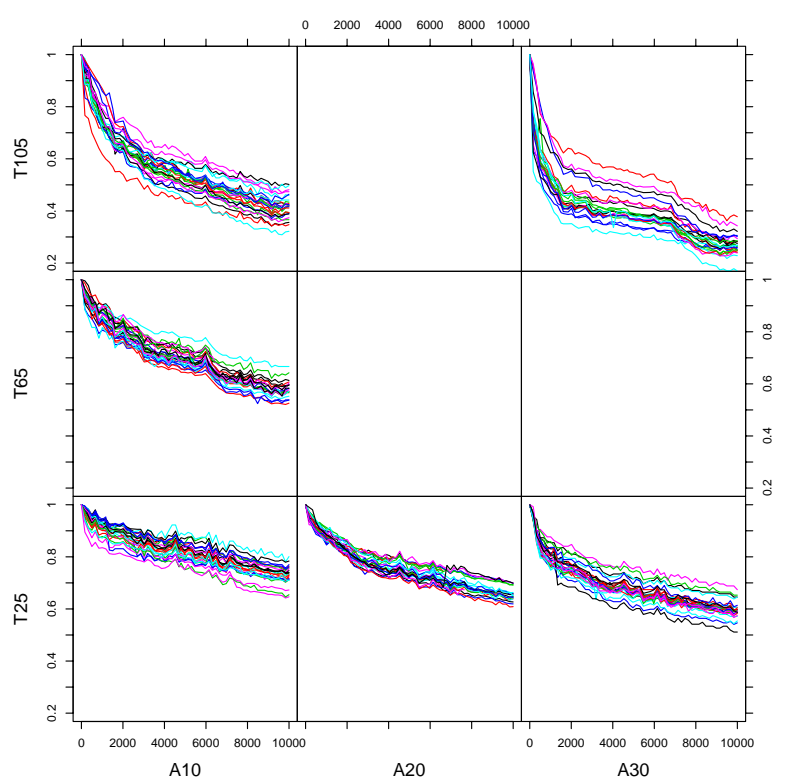

Figure 1: Accelerated degradation paths of LED data

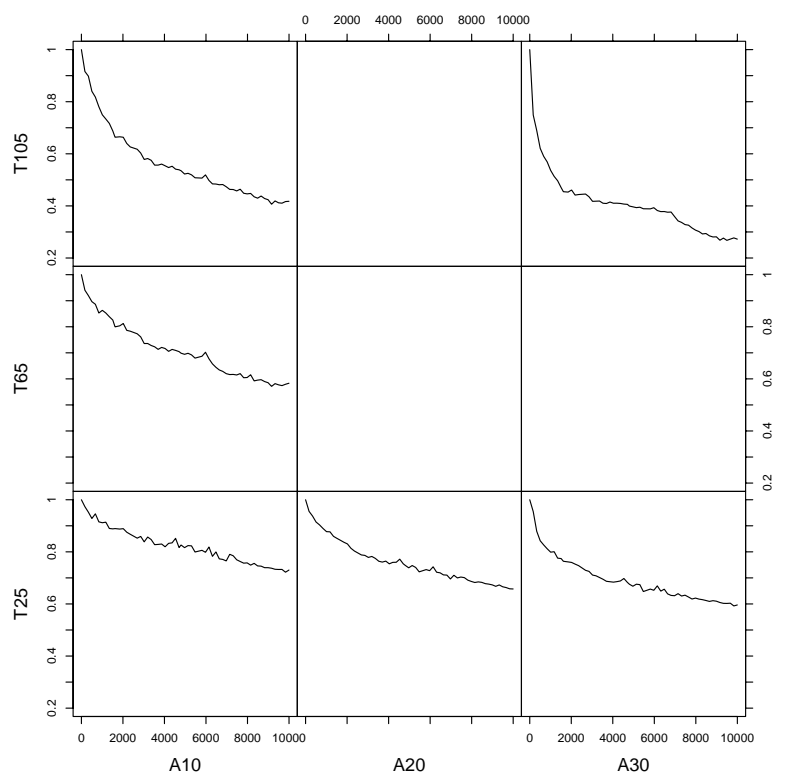

Figure 2: Sampled mean degradation paths of LED data

Figure 2 shows the plots of the sample mean degradation path, $\hat{M}_{i j}(t)$, for all six cells and Figure 3 plots $\log \left(-\log \hat{M}_{i j}(t)\right)$ against $\log (t)$. Roughly speaking, 


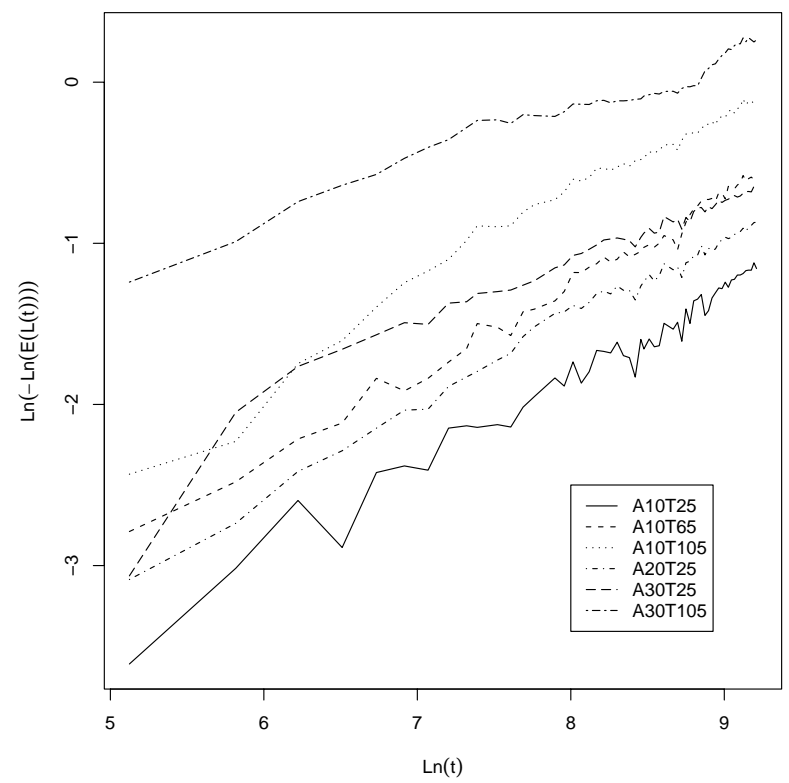

Figure 3: Transformation of sampled mean degradation paths of LED data

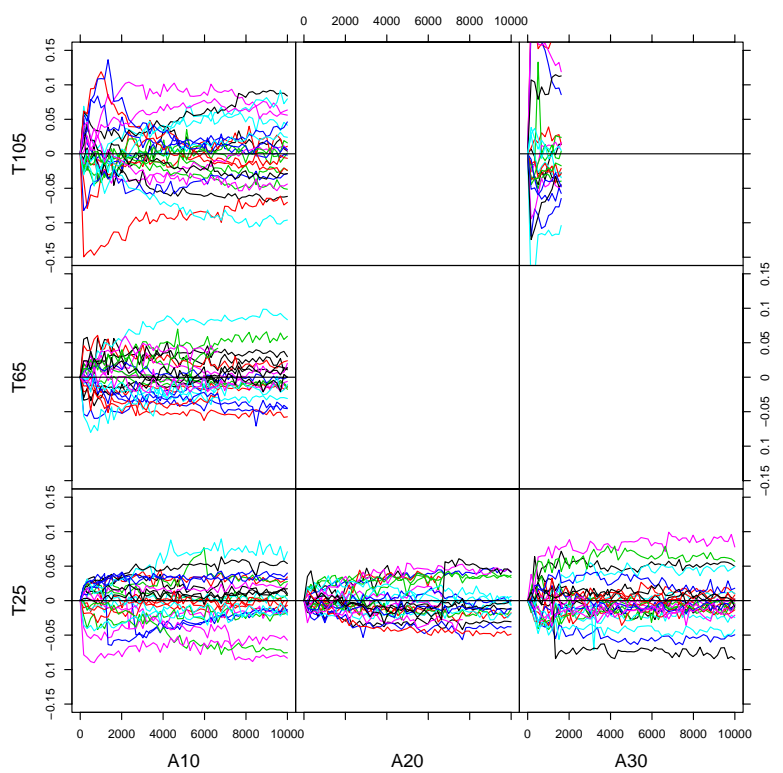

Figure 4: Residual plots of the LED degradation data

it shows a reasonable linear relationship between $\log \left(-\log \hat{M}_{i j}(t)\right)$ and $\log (t)$, except for the cell of $\left(30 \mathrm{~mA}, 105^{\circ} \mathrm{C}\right)$ has demonstrated a significantly over-stress 
pattern after 1620 hours. After confirming with the product engineers, the valid data for this cell is only restricted up to 1620 hours. The $R^{2}$ fitting for generalized Eyring law (of the truncated data) is 0.918. Hence, (5.2) and (5.3) are appropriate models for describing the mean degradation path and life-stress relationship of LED data, respectively.

Figure 4 shows the plots of $\hat{\varepsilon}_{i j k}\left(t_{l}\right)$ and it has an outward-opening funnel pattern. Figure 5 shows the plots of $\Delta \hat{\varepsilon}_{i j k}\left(t_{l}\right) / \Delta t_{l}$, where $\Delta t_{l}=t_{l}-t_{l-1}$. Except for few extreme cases, the means of all $\Delta \hat{\varepsilon}_{i j k}(t) / \Delta t$ are around 0 and their curve patterns are damped oscillation and decay over time. Note that $\Delta \hat{\varepsilon}(t) / \Delta t \approx$ $d \varepsilon(t) / d t$, for all $t$. Hence, (2.3) is an appropriate model for the error term of LED degradation path.

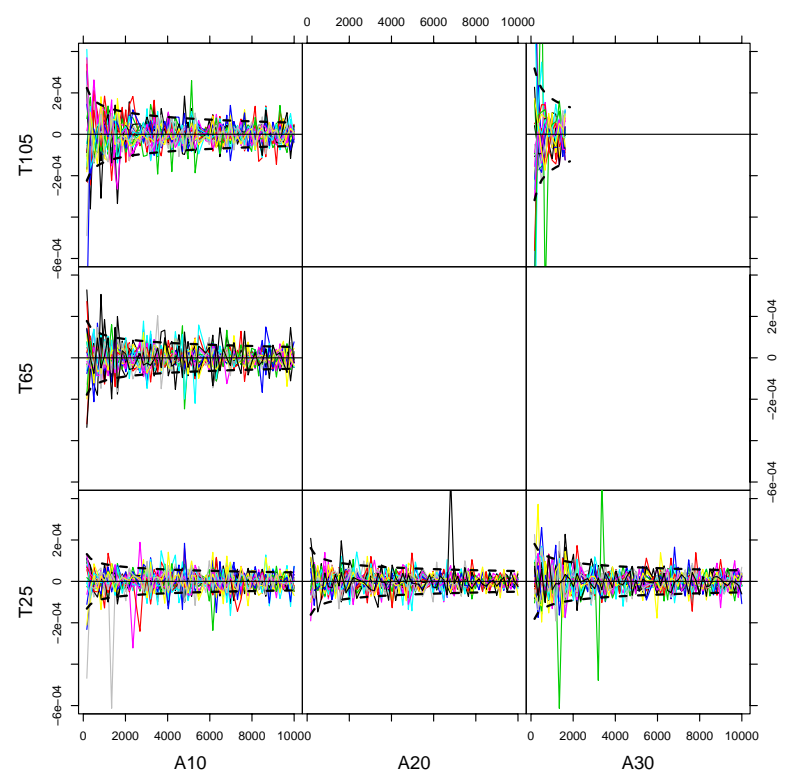

Figure 5: The derivative of the residual plots for the LED data

Now, let

$$
\hat{\Upsilon}_{i j}(t)=\frac{\hat{s}_{i j}^{2}(t)}{\hat{m}_{i j}(t)}, \forall t>0
$$

where

$$
\hat{m}_{i j}(t)=\frac{1}{n_{i j}} \sum_{k=1}^{n_{i j}}\left(\frac{\Delta L_{i j k}(t)}{\Delta t}\right)
$$

and

$$
\hat{s}_{i j}^{2}(t)=\frac{1}{n_{i j}-1} \sum_{k=1}^{n_{i j}}\left(\frac{\Delta L_{i j k}(t)}{\Delta t}-\hat{m}_{i j}(t)\right)^{2} .
$$


Then, by applying LOWESS (robust locally weighted regression and smoothing scatter plots) procedure proposed by Cleveland (1979), we conduct a scatter plot smoothing for $\hat{\Upsilon}_{i j}(t)$ and the results are shown in Figure 6 . It demonstrates that the assumption of (4.1) is reasonable for LED ADT data. Hence, we have

$$
L_{i j}(t)=\exp \left(-\eta_{i j} t^{\delta}\right)+\sigma B\left(1-\exp \left(-\eta_{i j} t^{\delta}\right)\right)
$$

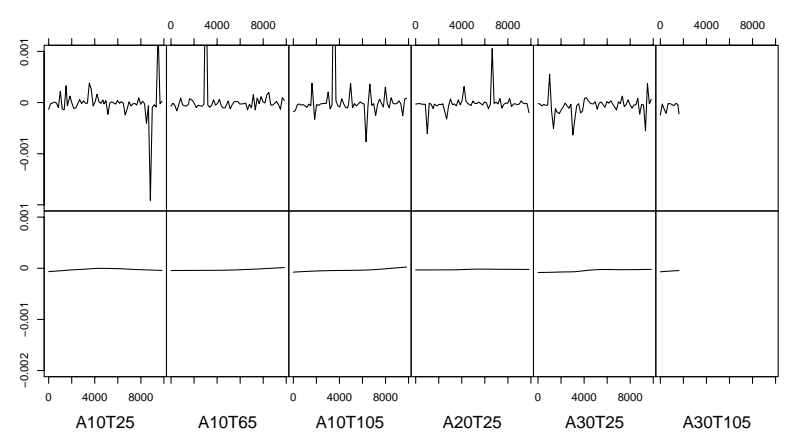

Figure 6: The plots of $\hat{\Upsilon}_{i j}(t)$ and its smoothing

Following from Section 5.1, the MLEs for unknown parameters can be solved numerically as follows:

$$
\left(\hat{\gamma_{0}}, \hat{\gamma_{1}}, \hat{\gamma_{2}}, \hat{\gamma_{3}}, \hat{\delta}, \hat{\sigma}\right)=(-3.8735,1.39,-1058.5766,-233.2695,0.5219,0.01286) .
$$

Now substitute the MLEs $\left(\hat{\gamma_{0}}, \hat{\gamma_{1}}, \hat{\gamma_{2}}, \hat{\gamma_{3}}\right)$ into (5.3), we obtain the result that $\hat{\eta}_{00}=0.00186$. Thus, under normal use stress $\left(7.5 \mathrm{~mA}, 20^{\circ} \mathrm{C}\right)$, the predicted $M T T F_{00}$ and $B 50_{00}$ are 84580.3 and 84452.0 , respectively. A bootstrap sampling technique is used to construct $95 \%$ confidence intervals for $M T T F_{00}$ and $B 50_{00}$. The results are [76254.8,92905.7] and [75152.4,93751.6], respectively.

\subsection{Performance of the proposed procedure}

In the LED data, for any cell of $\left(A_{i}, T_{j}\right)$ with a larger $\omega$, the sample MTTF can be obtained directly via the sample mean of the first passage time of its degradation path that hits $\omega$. In the meantime, the predicted MTTF of the cell of $\left(A_{i}, T_{j}\right)$ can be obtained by replacing $\left(\eta_{i j}, \delta, \sigma\right)$ in $(5.4)$ by $\left(\hat{\eta}_{i j}, \hat{\delta}, \hat{\sigma}\right)$, respectively. Hence, the performance of the proposed procedure can be observed via its relative prediction error between the sample MTTF and its predicted MTTF. Under $0.6 \leq \omega \leq 0.8$, Table 1 shows the predicted MTTF (we denote our proposed procedure as TP method), the sample MTTF and their relative prediction error for all six combinations of $\left(A_{i}, T_{j}\right)$. For example, when $\omega=0.7$, the sample MTTF for the cell of $\left(20 \mathrm{~mA}, 25^{\circ} \mathrm{C}\right)$ is 7121.5 hours and its predicted 


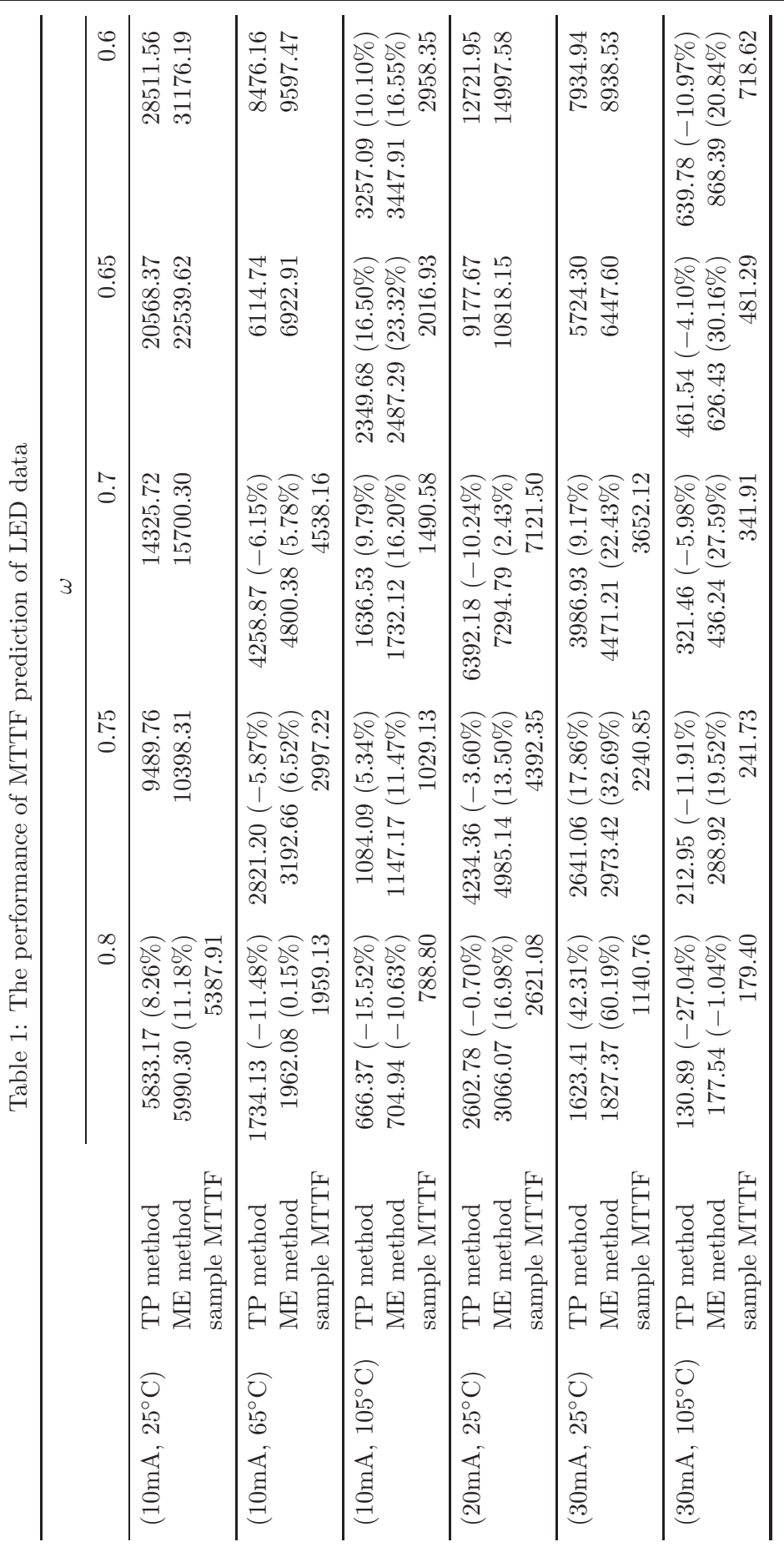




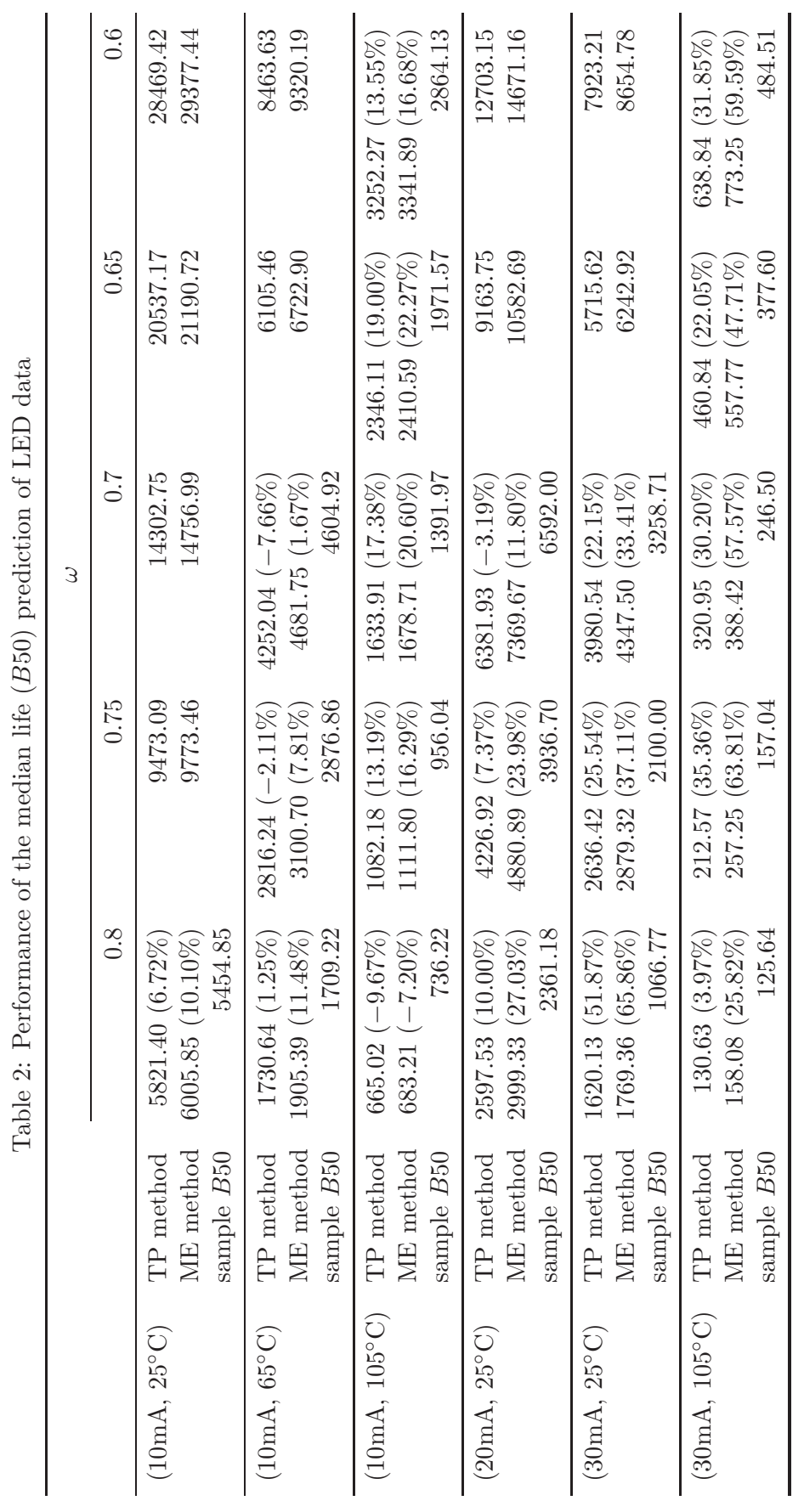


MTTF is 6392.18 hours. The relative prediction error is $-10.24 \%$. Except for one extreme cases in $\left(30 \mathrm{~mA}, 25^{\circ} \mathrm{C}\right)$ with $(\omega=0.8,42.31 \%)$, and one case in $\left(30 \mathrm{~mA}, 105^{\circ} \mathrm{C}\right)$ with $(\omega=0.8,-27.04 \%)$, all the relative prediction errors are significantly less than $\pm 20 \%$. It demonstrates that (5.4) provides a good lifetime prediction for LED data.

Similarly, under $0.6 \leq \omega \leq 0.8$, Table 2 shows the predicted $B 50$ (again, we denote it as TP method), the sample $B 50$ and their relative prediction error for all six combinations of $\left(A_{i}, T_{j}\right)$. Again, for $0.6 \leq \omega \leq 0.8$, we use (5.5) to assess the product's $B 50$ of all six cells in the LED data. Roughly speaking, the proposed procedure of (5.5) has a good performance in its $B 50$ prediction except for the cells with an extreme high electric current (say $30 \mathrm{~mA}$ ).

\subsection{Comparison with mixed effect model}

As we mentioned earlier that the mixed effects (ME) model is a well-known approach for the degradation data. Hence, we would like to compare the predicted performance between our proposed method and ME model (such as Meeker et al., 1998). Note that ME model has taken the unit-to-unit variation of test units into consideration. Hence, the ME model for the LED data is completely the same as (5.1-5.3), except for considering that $\left(\gamma_{0}, \gamma_{1}, \gamma_{2}, \gamma_{3}\right)$ follows a multivariate normal distribution with $N_{4}\left(\gamma^{*}, \Sigma^{*}\right)$ and $\varepsilon_{i j k}(t)$ follows an iid $N\left(0, \sigma_{\varepsilon}^{2}\right)$. From Figure 3 , we can reasonably assume that $\delta$ is a fixed, unknown parameter. Now, set

$\mathbf{a}^{T}=\left(1 \ln A_{i} \frac{1}{273.15+T_{j}} \frac{\ln A_{i}}{273.15+T_{j}}\right)$. Then $\ln \eta_{i j}$ follows a normal distribution with $N\left(\mathbf{a}^{T} \gamma^{*}, \mathbf{a}^{T} \Sigma^{*} \mathbf{a}\right)$. The cdf of lifetime distribution with the mixed effects model can be derived as follows:

$$
F_{M E}(t)=1-\Phi\left(\frac{\ln (-\ln \omega)-\delta \ln t-\mathbf{a}^{T} \gamma^{*}}{\sqrt{\mathbf{a}^{T} \Sigma^{*} \mathbf{a}}}\right) .
$$

Hence, we have

$$
M T T F_{M E}=\int_{0}^{\infty}\left(1-F_{M E}(t)\right) d t
$$

and

$$
B 50_{M E}=\left(\frac{-\ln \omega}{\eta}\right)^{1 / \delta}
$$

Now, return to the LED data again, the MLEs of all unknown parameters in the mixed effects model are $\hat{\delta}=0.5231, \hat{\sigma}_{\varepsilon}=0.0310, \hat{\gamma}^{*}=(-3.8782,1.3864,-1059.8324,-233.4621)^{T}$ and

$$
\hat{\Sigma}^{*}=\left(\begin{array}{rrrr}
0.0030 & 0.0010 & 0.6639 & 0.0881 \\
0.0010 & 0.0004 & 0.2257 & 0.0309 \\
0.6639 & 0.2257 & 148.3043 & 19.5429 \\
0.0881 & 0.0309 & 19.5429 & 2.6643
\end{array}\right) \text {. }
$$


Similar to Section 6.1, the performance of the mixed effects model can be addressed via its relative prediction errors between the sample MTTF and its predicted MTTF. Under $0.6 \leq \omega \leq 0.8$, Table 1 also shows the predicted MTTF and their relative prediction errors of the ME method for all six combinations of $\left(A_{i}, T_{j}\right)$. For example, under $\omega=0.7$, and $\left(10 \mathrm{~mA}, 65^{\circ} \mathrm{C}\right)$, the relative prediction errors for our method and ME method are $-6.15 \%$ and $5.78 \%$, respectively. However, under $\omega=0.7$, and $\left(30 \mathrm{~mA}, 25^{\circ} \mathrm{C}\right)$, the relative prediction errors for our method and ME method are $9.17 \%$ and $22.43 \%$, respectively. It did not demonstrate that which one has overall better prediction. However, for each cell of $\left(A_{i}, T_{j}\right)$, it demonstrates a trend that prediction errors of our method are smaller than that of ME method as $\omega$ decreases. Hence, it implies that our method may have smaller prediction error when $\omega$ is near to 0.50. Similarly, under $0.6 \leq \omega \leq 0.8$, Table 2 also shows the predicted $B 50$ and their relative prediction errors of ME method for all six combinations of $\left(A_{i}, T_{j}\right)$. It demonstrates that our method of (5.5) has a significantly better performance than that of $\mathrm{ME}$ model except for two cases: $(\omega=0.7)$ with $\left(10 \mathrm{~mA}, 65^{\circ} \mathrm{C}\right)$ and $(\omega=0.8)$ with $\left(10 \mathrm{~mA}, 105^{\circ} \mathrm{C}\right)$. In addition, it also demonstrates a trend that our method has a smaller prediction error as $\omega$ decreases.

\section{Conclusion}

In this paper, we introduced a stochastic diffusion process to model the product's degradation path and two approximate formulas for assessing the product's MTTF and B50 are also derived under a reasonable assumption on its degradation model. Finally, a LED data is used to illustrate the proposed procedure and prediction performance between our procedure with the ME model is also addressed. From Section 6.2, except for few cases, it demonstrates that our proposed procedure has better prediction performance (in its MTTF and B50 prediction) than the ME model. In addition, our approach provides the reliability analysts with the explicit expressions for the product's MTTF and B50, which can not be obtained by using the conventional mixed effects formulation.

\section{Acknowledgements}

This work was partially supported by the National Science Council (NSC) of Taiwan-ROC. The authors are indebted to the Editor of JDS, Dr. M. T. Chao and Prof. W. Q. Meeker for their valuable comments which made the paper more readable. Besides, we thank Mr. Tseng, M. and LITEON Corporation for kindly providing the LED data set. 


\section{References}

Bae, S. J. and Kvam, P. H. (2004). A Nonlinear Random-Coefficients Model for Degradation Testing. Technometrics 46, 460-469.

Chang, D. S. (1992). Analysis of accelerated degradation data in a two-way design. Reliability Engineering and System Safety 39, 65-69.

Chao, M. T. (1999). Degradation analysis and related topics: Some thoughts and a review. The Proceedings of the National Science Council, Part A. 23, 555-566.

Chhikara, R. S. and Folks, L. (1989). The Inverse Gaussain Distribution. Theory, Methodology, and Applications. Marcel Dekker.

Cleveland, W. S. (1979). Robust locally weighted regression and smoothing scatterplots. Journal of the American Statistical Association 74, 829-836.

Di Nardo, E., Nobile, A. G., Pirozzi, E. and Ricciardi, L. M. (2001). A computational approach to first-passage-time problems for Gauss-Markov processes. Advances in Applied Probability 33, 453-482.

Doksum, K. A. and Hóyland, A. (1992). Model for variable-stress accelerated life testing experiments based on Wiener processes and the inverse Gaussian distribution. Technometrics 34, 74-82.

Fukuda, M. (1991). Reliability and Degradation of Semiconductor Lasers and LEDs. Artech House.

Hoel, P. G., Port, S. C. and Stone, C. J. (1972). Introduction to Stochastic Processes. Waveland Press.

Lawless, J. and Crowder, M. (2004). Covariates and Random Effects in a Gamma Process Model with Application to Degradation and Failure. Lifetime Data Analysis 10, 213-227.

Lu, J. C., Park, J. and Yang, Q. (1997). Statistical inference of a time to failure distribution derived from linear degradation data. Technometrics 39, 391-400.

Mehr, C. B. and McFadden, J. A. (1965). Certain properties of Gaussian processes and their first-passage times. Journal of the Royal Statistical Society. Series B 27, 505-522.

Meeker, W. Q. and Escobar, L. A. (1998). Statistical Methods for Reliability Data. John Wiley \& Sons.

Meeker, W. Q., Escobar, L. A. and Lu, C. J. (1998). Accelerated degradation test: modeling and analysis. Technometrics 40, 89-99.

Nelson, W. (1990). Accelerated Testing: Statistical Models, Test Plans, and Data Analysis. John Wiley \& Sons.

Patel, R. C. (1965). Estimates of parameters of truncated inverse Gaussian distribution. Annals of the Institute of Statistical Mathematics 17, 29-33. 
$\emptyset$ ksendal, B. (2003). Stochastic Differential Equations: An Introduction with Applications. Sixth Edition, Springer.

Shiau, J. -J. H. and Lin, H. H. (1999). Analyzing accelerated degradation data by nonparametric regression. IEEE Transactions on Reliability 48, 149-158.

Tseng, S. T., Hamada, M. S. and Chiao, C. H. (1995). Using degradation data from a fractional factorial experiment to improve fluorescent lamp reliability. Journal of Quality Technology 27, 363-369.

Tseng, S. T. and Peng, C. Y. (2004). Optimal burn-in policy by using integrated Wiener process. IIE Transactions 36, 1161-1170.

Tseng, S. T., Tang, J. and Ku, I. H. (2003). Determination of burn-in parameters and residuals life of highly reliable products. Naval Research Logistics 50, 1-14.

Whitmore, G. A. and Yalovsky, M. (1978). A normalizing logarithmic transformation for inverse Gaussian random variables. Technometrics 20, 207-208.

Yu, H. F. and Tseng, S. T. (1998). On-Line procedure for terminating an accelerated degradation test. Statistica Sinica 8, 207-220.

Yu, H. F. and Tseng, S. T. (2002). Designing a screening experiment for highly reliable products. Naval Research Logistics 49, 514-526.

Received March 6, 2006; accepted May 22, 2006.

\author{
Sheng-Tsaing Tseng \\ Institute of Statistics \\ National Tsing-Hua University \\ Hsin-chu, Taiwan 30013 \\ sttseng@stat.nthu.edu.tw \\ Chien-Yu Peng \\ Institute of Statistics \\ National Tsing-Hua University \\ Hsin-chu, Taiwan 30013 \\ chienyu@stat.sinica.edu.tw
}

